\title{
Use of Color Flow Doppler Ultrasonography to Increase the Safety of Intrathecal Drug Delivery System Refills
}

\author{
Ankur D. Mehta, DO', Mitchell P. Engle, MD², and Vishal Bansal, MD³
}

Intrathecal drug delivery systems are effective tools in the treatment of chronic pain or spasticity. Using intrathecal drug delivery systems, drugs are directly infused into the cerebrospinal fluid and gain access to critical receptors in the central nervous system. This delivery method allows for the use of markedly reduced doses of medications to produce the same effects as seen with higher orally administered doses. Reduced dosing produces fewer side effects, creating a more favorable treatment course for patients already suffering from chronic pain or spasticity. Complications with intrathecal drug delivery systems can occur during both the implantation process and the postoperative maintenance of the device. The most common procedure carried out during postoperative maintenance of these devices is the intrathecal pump refill. This procedure must be performed by experienced practitioners in a systematic way in order to prevent complications. The most dreaded of these complications is inadvertent injection of medication into the subcutaneous space as opposed to into the device, commonly referred to as a "pocket fill." This paper describes and recommends the routine use of color flow doppler ultrasonography as an easy, safe, and effective tool to reduce the chance of a catastrophic "pocket fill." Routine utilization of live color flow ultrasound guidance during pump refills allows the practitioner to carefully document flow of medication into the pump under the silicone septum. It also allows for demonstration of no leakage from, or fluid increase superficial to, the septum. Additionally, 2 ultrasound images are obtained to document color flow deep to the septum and no color flow/leak/fluid superficial to the septum. Although this complication is rare, it can lead to severe complications including oversedation, respiratory depression, and death. As such, we believe that the utilization of color phase doppler ultrasonography could further reduce this incidence.

Key words: Analgesics, opioids, chronic pain, drug overdose, infusion pumps, implantable, injections, spinal, medication errors, spasticity, ultrasonics, methods, ultrasonography, interventional
Intrathecal drug delivery systems (IDDS) are an alternative and effective means of delivering opioids and other medications to patients with refractory

From : ${ }^{1} \mathrm{TX}$ Spine \& Joint Clinic, Houston, TX; ${ }^{2}$ Institute of Precision Pain Medicine, Corpus Christi, TX; ${ }^{3}$ Caribbean Medical University, Willemstad, Curacao

Author for correspondence: Ankur D. Mehta, DO

Address: TX Spine \& Joint Clinic, 8830 Long Point Road, Suite 502, Houston, TX 77055

E-mail:MehtaDO@gmail.com

Disclaimer: There was no external funding in the preparation of this manuscript. Conflict of interest: Each author certifies that he or she, or a member of his or her immediate family, has no commercial association (i.e., consultancies, stock ownership, equity interest, patent/licensing arrangements, etc.) that might pose a conflict of interest in connection with the submitted manuscript. chronic pain or spasticity. These select groups of patients with chronic pain have generally failed first- and second-line treatments, failed to achieve adequate reduction in pain ratings, and/or are not able to tolerate optimal dosing. The introduction of IDDS has allowed for medications to be delivered directly into the intrathecal space. This therapeutic modality has allowed for optimal and effective analgesia with reduced doses of medications and reduced side effects (1). The smaller doses that are used allow for a reduction in side effects that are routinely seen with systemic use $(2,3)$. Medications that are delivered through the intrathecal sac gain direct access to the cerebrospinal fluid and avoid crossing the blood-brain barrier. The dorsal horns of the spinal cord within the 
intrathecal space are a vital location for pain processing with a high density of pain-related receptors (4). In addition to the advantage of reduced dosing, direct delivery also allows for a more prompt and optimal response (2).

However, the use of IDDS can lead to drug-, procedure-, and device-related complications (5). Some of the major complications and adverse outcomes arise from the implantation process and the postoperative maintenance of IDDS (5). Postoperative maintenance entails intrathecal pump refills as well as pump and catheter replacements. The most frequent maintenance procedure with the device is refilling the pump reservoir. This involves using a sterile process to locate and gain access to the pump port, emptying any residual medication, and then refilling the device with new medication. Specific complications arising during pump refills include inability to access the pump reservoir, infections, seroma formation, and inadvertent injection of medications outside the pump (6).

The inadvertent injection of medications outside of the pump reservoir into the surrounding subcutaneous space is commonly referred to as a pump "pocket fill" $(7,8)$. Pocket fills can lead to a multitude of complications relating to drug overdose of the specific medication(s) being used. This includes and is not limited to oversedation, respiratory depression, and death (9). A clinical suspicion of a pocket fill should come to attention when a patient reports swelling, stinging, or tingling at the injection site during the refill, or any overdose symptoms pertaining to the medication(s) being used $(7,10)$.

Current safety precautions employed to help mitigate the risks of pocket fills include contacting the back of the pump reservoir with the needle, serial aspirations, and confirmation of volume from expected and actual levels $(7,11)$. Recommendations are also made in current literature to employ the use of fluoroscopy and ultrasound during difficult refill procedures $(10,12)$.

We propose a novel and unique method of routinely using ultrasound-guided imaging with color flow doppler to complete safe and precise pump refills. Described herein, we outline how the use of routine ultrasound-guided imaging with color doppler allows for precise extraction of residual medication and the introduction of new medication into the pump reservoir. Routine use of ultrasound will also allow for risk mitigation of multiple needle sticks when trying to contact the pump reservoir.

\section{Description of Technique}

The region of the intrathecal pump is prepped and draped in a sterile fashion. The silicone septum is identified, and using ultrasound guidance, a noncoring 22-gauge Huber needle is advanced into the pump's refill port until a firm "end-feel" is felt. The current medication is then aspirated, and volume is confirmed prior to wasting. The new medication is then injected through a 0.2-micron filter under sterile conditions. Live color flow ultrasound guidance is used during this injection to carefully document the flow of medication into the pump under the silicone septum. It also allows for demonstration of no leakage from, or fluid increase superficial to, the septum. Two ultrasound images are generally obtained showing color flow deep to the septum and no color flow/ leakage/fluid superficial to the septum (Figs. 1,2). The needle is then withdrawn and sterile dressings are applied.

\section{DISCUSSION}

Ultrasounds use probes that generate pulses and record the characteristics of echoes received from the structures being examined. The addition of color doppler allows visual representation of color-guided velocity information that is superimposed on greyscale imaging of the tissue and implanted device (13). The echoes produced from the device and anatomical tissue stay the same from pulse to pulse and the echoes from moving fluid produce varying differences that are displayed as a frequency.

The doppler feature can represent flow that is going towards the probe (red) and away from the probe (blue) through simple color schematics; it can also display intensity of flow with brighter shades indicating faster flow and darker shades indicating slower flow (13). Doppler imaging is a common feature on ultrasound machines and can prove to be an important safety measure while performing pump refills. 


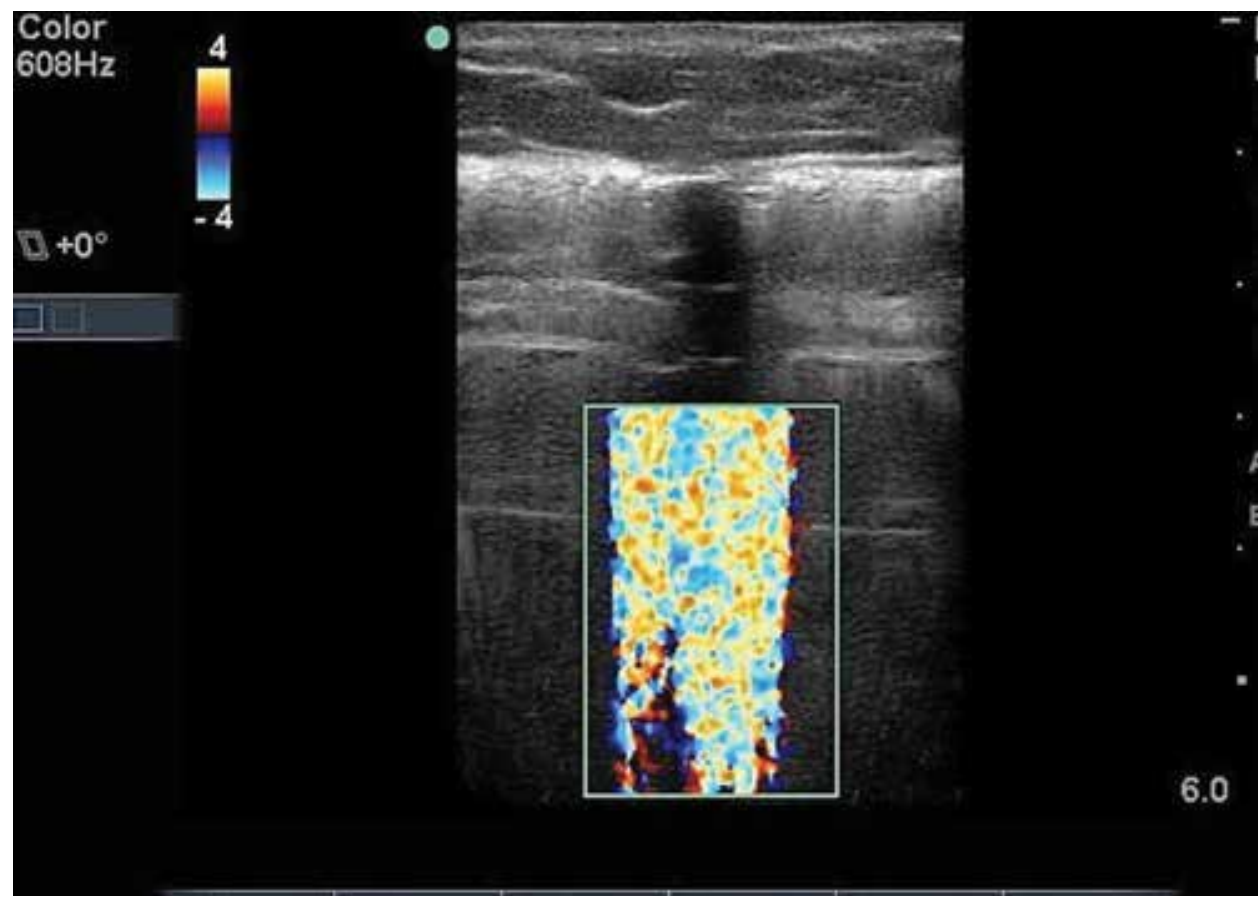

Fig. 1. Ultrasound images with color flow confirms flow of medications into the pump reservoir.

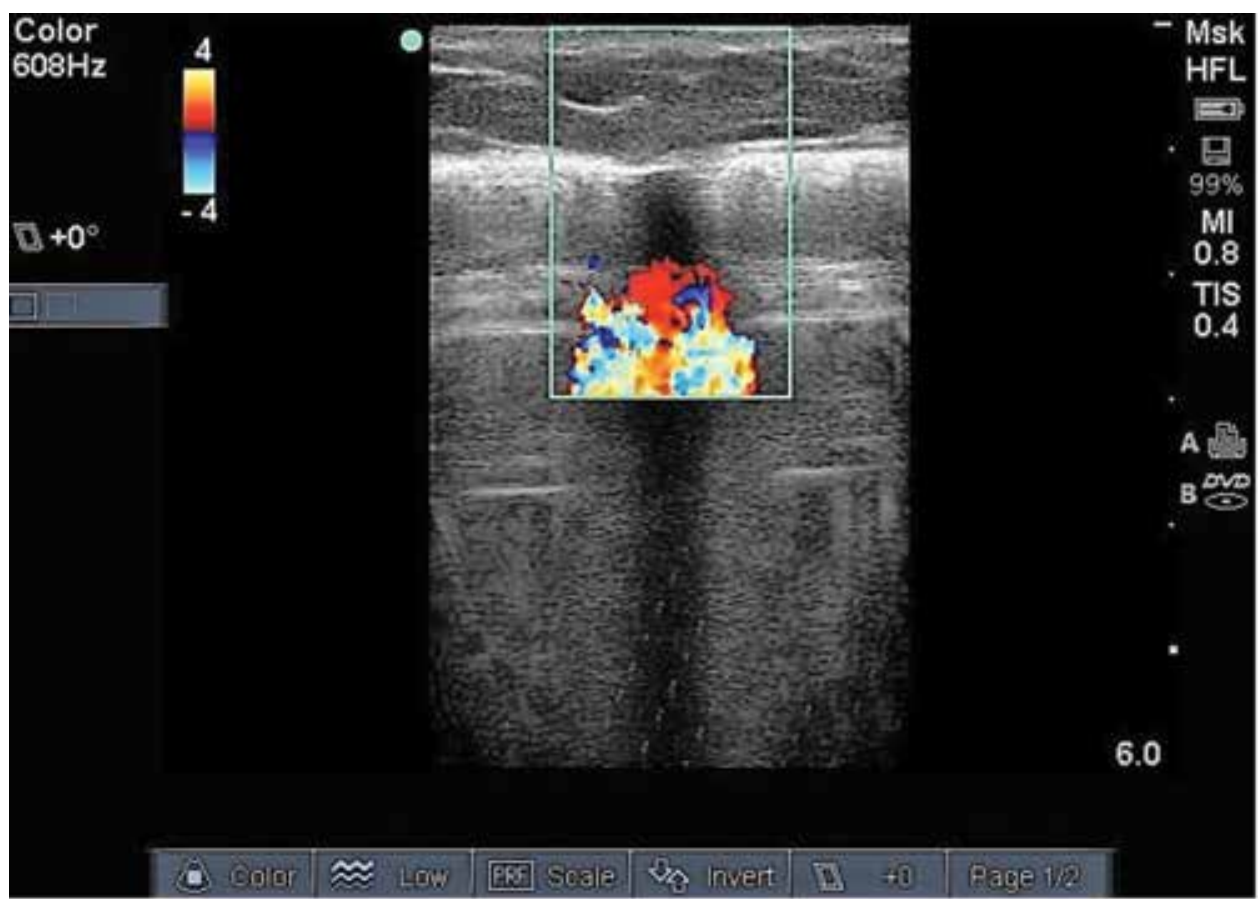

Fig. 2. Ultrasound image confirming no flow of medications outside of the septum in intrathecal pain pump. 


\section{CONCLUSION}

Ultrasonography continues to emerge as a valuable tool in all fields of medicine. Herein we describe the use of color flow ultrasonography to increase the safety of intrathecal pump refills. The use of this procedure allows the practitioner to directly visualize the injection of the refill medication deep to the septum, likely markedly increasing the safety of the refill procedure. The Polyanalgesic Consensus Conference (PACC) performed an exhaustive review of current literature to compile a report detailing methods and practices to mitigate risks associated with IDDS systems (14). Within these recommendations, there is emphasis on current literature that advocates for ultrasound guidance when the pump refill port is difficult to visualize and demonstrates the benefits of doppler for direct visualization of medication in the pump (15).

A study by Coffey et al (15) that reviewed 557 evaluations of IDDS systems in non-cancer patients from 1998 to 2007 revealed 88 deaths within 3 days of a pump implant, refill, replacement, reprogramming, dose change, or catheter revision. Amongst the 88 reported deaths, $28(32 \%)$ were attributed to pump refills (15). In January 2011, Medtronic reported 8 deaths and 270 events from May 1996 to September 2010 that required medical intervention due to incidental pocket fills. The incidence of pump pocket refills is approximated at one per 10,000 refills $(0.01 \%)$ as reported by Medtronic (16). Although this complication is rare, it has a significant rate of morbidity and mortality when it does occur. As such, we believe that the utilization of color phase doppler ultrasonography could further reduce this incidence. Ultrasound continues to be one of the most commonly used imaging modalities in health care. Ultrasound imaging is portable, lacks any radiation risk, and is inexpensive when compared to other imaging options (17). In the setting of interventional pain management, health care providers generally have experience with ultrasound technology with color doppler. In addition, ultrasound imaging is optimal and effective when refilling a patient with an abnormal body habitus or hard-to-access IDDS (12). Furthermore, we advocate for the routine use of ultrasound with color doppler for all refill procedures and not only during certain complicated procedures. 


\section{REFERENCES}

1. Christo $P$, Bottros $M$. Current perspectives on intrathecal drug delivery. J Pain Res 2014; 7:615-626.

2. Bhatia G, Lau M, Koury K, Gulur P. Intrathecal drug delivery (ITDD) systems for cancer pain. FroooRes 2013; 2:96.

3. Duarte R, Raphael J, Eldabe S. Intrathecal drug delivery for the management of pain and spasticity in adults: An executive summary of the British Pain Society's recommendations for best clinical practice. Br J Pain 2015; 10:67-69.

4. Kim PS, Li S, Deer TR, Wallace MS, Staats P. Intrathecal drug delivery systems. In: Manchikanti L, Kaye A, Falco F, Hirsch J (eds). Essentials of Interventional Techniques in Managing Chronic Pain. Basel, Switzerland, Springer International Publishing, 2018: pp. 671-681.

5. Follett K, Burchiel K, Deer T, et al. Prevention of intrathecal drug delivery catheter-related complications. Neuromodulation 2003; 6:32-41.

6. Gritsenko K, Carullo V, Deer TR. Complications of intrathecal drug delivery. In: Deer TR, Pope J (eds). Atlas of Implantable Therapies for Pain Management. New York, Springer, 2016: pp. 301-312.

7. Maino P, Perez RS, Koetsier E. Intrathecal pump refills, pocket fills, and symptoms of drug overdose: A prospective, observational study comparing the injected drug volume vs. the drug volume effectively measured inside the pump. Neuromodulation 2017; 20:733-739.8.

8. Peccora CD, Ross EL, Hanna GM. Aberrant intrathecal pump refill. Reg Anesth Pain Med 2013; 38:544-546.

9. Naumann C, Erdine S, Koulousakis A, Van Buyten JP, Schuchard M. Drug adverse events and system complications of intrathecal opioid delivery for pain: Origins, detection, manifestations, and management. Neuromodulation 1999; 2:92-107.
10. Timothy DR, Levy R, Prager J, et al. Polyanalgesic Consensus Conference-2012: Recommendations to reduce morbidity and mortality in intrathecal drug delivery in the treatment of chronic pain. Neuromodulation 2012; 15:467-482.

11. Greher M, Eichenberger U, Gustorff B. Sonographic localization of an implanted infusion pump injection port: Another useful application of ultrasound in pain medicine. Anesthesiology 2005; 102:243.

12. Evans DH, Jensen JA, Nielsen MB. Ultrasonic colour Doppler imaging. Interface Focus 2011; 1:490-502.

13. Deane C. Doppler ultrasound: Principles and practice. Sonoworld Web site. https://sonoworld.com/client/fetus/html/doppler/ capitulos-html/chapter_01.htm. Date Published 01/01/2002. Date Accessed 01/20/2019.

14. Deer TR, Pope JE, Hayek SM, et al. The Polyanalgesic Consensus Conference (PACC): Recommendations for intrathecal drug delivery: Guidance for improving safety and mitigating risks. Neuromodulation 2017; 20:155-176.

15. Coffey RJ, Owens ML, Broste SK, et al. Mortality associated with implantation and management of intrathecal opioid drug infusion systems to treat noncancer pain. Anesthesiology 2009; 111:881-891.

16. FDA classifies labeling corrections related to occurrence of pocket fills during a SynchroMed $囚$ implantable infusion pump refill as a Class I Recall [news release]. Minneapolis, MN: Medtronic; February 16, 2011. http://newsroom.medtronic.com/phoenix. zhtml?c=251324\&p=irol-newsArticle\&ID=1773319\&highlight= . Date Accessed 01/01/2019.

17. Chan V, Perlas A. Basics of ultrasound imaging. In: Narouze S (ed). Atlas of Ultrasound-Guided Procedures in Interventional Pain Management. New York, Springer, 2011: pp. 13-19. 
